\title{
Herencias y retos del conocimiento en América Latina
}

RAQUEL SOSA ELÍZAGA

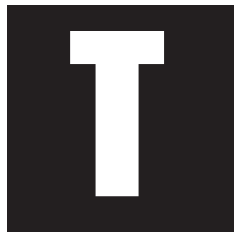

oda empresa de conocimiento se enfrenta a determinaciones que condicionan sus posibilidades y alcances. El interés por abordar uno u otro tema, explicable por el horizonte de visibilidad de quien aspira a conocer, suele estar cercado por presiones variadas, entre las que se señalan las limitaciones profesionales, la legitimación de conceptos y categorías producidos por autores de cierto reconocimiento, la accesibilidad a fuentes $y$, desde luego, la constitución de corrientes que favorecen visiones políticamente orientadas.

Si a dichas presiones agregamos el hecho de que la mayor parte de las investigaciones que se emprenden tienen un objetivo de inicio limitado, ya que suelen servir en las universidades para el fin específico de aprobar un curso, conseguir fondos para becas o apoyos de investigación, o aún mostrar evidencias empíricas para sustentar hipótesis sugeridas o impuestas desde fuera del propio estudio, tenemos un cuadro básico para entender las razones por las cuales suele pasarse por alto la responsabilidad social que implica la orientación del trabajo de investigación.

No obstante, es posible y necesario realizar evaluaciones de la bibliografía que sobre diversos temas existen a disposición del investiga-

* Secretaria de Cultura del Gobierno del Distrito Federal. Doctora en Historia de México por la Universidad Nacional Autónoma de México. 
dor contemporáneo. Aún sin esforzarse demasiado, un observador cuidadoso puede constatar la existencia de estudios orientados por las corrientes dominantes en cada periodo, sin que en ellos se plantee la pertinencia del estudio emprendido, su vinculación con políticas de investigación orientadas por fines extra académicos, su posible impacto o alcance en la sociedad a la que se hace objeto de estudio. Mucho menos se encuentra, en la mayor parte, asomo de cuestionamiento sobre los temas no tratados, las perspectivas disciplinarias ausentes, la orientación y sentido mismo de la investigación, o hasta la historia de los propios temas de interés.

Qué y porqué se estudia, desde dónde se sitúa un investigador, de qué elementos dispone, cómo evalúa sus propias fuentes de conocimiento, en qué medida aporta un conocimiento novedoso, o simplemente reproduce conocimientos socialmente validados, cómo integra (o por qué razón deja de lado) otros campos de conocimiento que puedan servir a su propio objeto, constituyen formalidades usualmente obligadas en tesis de grado y que se conocen como "marco teórico". Poco aportan, en general, en el desarrollo mismo de esos trabajos académicos, pero, en todo caso, no se cuenta siquiera con ellas para evaluar el sentido, resultados e impacto de la investigación social en general.

En América Latina, el estudio de las determinaciones sociales del conocimiento, o lo que pudiera llamarse una sociología de los modos de pensar, es poco frecuente. Podemos, sin temor a equivocarnos, asumir que se trata de un campo prácticamente inexplorado. Aportaciones extraordinarias, como las de Sergio Bagú, son solitarios ejemplos de la que debiera ser una actividad tan rica como la del conocimiento social mismo ${ }^{1}$ (Bagú, 1970). En la dirección sugerida por él, diversos autores han explo-

\footnotetext{
1 "Privilegios y ausencias de determinación: periodos, temas, aspectos o especialidades; separaciones tradicionales entre disciplinas" en Coordinación de Humanidades/Facultad de Filosofía y Letras de la UNAM, Balance y perspectiva de los estudios latinoamericanos, México, 1985; "Evocación sobre la capacidad de crear ideas nuevas", Estudios Latinoamericanos no. 2, enero-junio de 1987; "Ciencias sociales en América Latina: observaciones sobre una tendencia generalizada", Estudios Latinoamericanos nos 6-7, enero-diciembre de 1989.
} 
rado la evolución del pensamiento latinoamericano. ${ }^{2}$ Exponen, además de las presiones que sobre la investigación se ejercen en general sobre el conocimiento social, las que se agregan en nuestra región debido a la escasa capacidad de difusión del conocimiento producido por nuestros centros de educación superior; la incipiente formación de redes de investigación sobre problemas comunes de diversa índole; las continuas presiones extra académicas por la adopción de definiciones ideológicas y conceptuales rígidas, usualmente asociadas al financiamiento de la investigación; y el carácter frecuentemente subordinado de intelectuales y académicos a la investigación social que se desarrolla en Europa y los Estados Unidos de América, por razones de status, protagonismo y búsqueda de trascendencia, ajenas a las búsquedas o necesidades de conocimiento de sus propias sociedades.

Esta situación contrasta no sólo con la riqueza y velocidad en que ocurren cambios sociales en la región, sino incluso con el número de estudios anualmente producido. El panorama del conocimiento social en América Latina es mucho más vasto, que lo que abarcan los limitados intentos por reconstruir su sentido y orientación, y considerablemente inferior a la ocurrencia de transformaciones en la realidad social. Ello afecta, indudablemente, nuestra posibilidad de reflexionar, acumular, resumir problemas significativos, en relación a las aportaciones y limitaciones del pensamiento en nuestras sociedades.

¿Cuántos se han preguntado, por ejemplo, sobre qué aportó el estudio de las etapas y formas del capitalismo de los años sesenta y setenta al

\footnotetext{
2 Agustín Cueva, "El desarrollo de nuestras ciencias sociales en el último período" en Teoría social y procesos políticos en América Latina, México, Edicol, 1979; AC, "Sobre exilios y reinos (Notas críticas sobre la evolución de la sociología sudamericana)" Estudios Latinoamericanos no. 4, enero-junio de 1988; Pablo González Casanova, "Las ciencias sociales en América Latina" en Balance y perspectiva, cit.; Hugo Zemelman, "Hacia una reflexión sobre las ciencias sociales en América Latina", Estudios Latinoamericanos nos. 6-7, enero-diciembre 1989; R. S."Evolución de las ciencias sociales en América Latina", Estudios Latinoamericanos Nueva Epoca no. 1, enero-junio 1994; R.S., "América Latina: ciencias sociales y sociedad hacia el siglo XXI", Estudios Latinoamericanos nos. 12-13, julio 1999-junio 2000.
} 
conocimiento de las transformaciones económicas y sociales actuales en la región? ¿Qué tránsito explica la evolución del conocimiento de las formas de dominación, la lucha por la hegemonía, a la consideración exclusiva de la democracia como eje explicativo de las relaciones políticas? ¿Qué investigaciones se han realizado a propósito de la sustitución de la temática del desarrollo por la del crecimiento económico? ¿Conocen los investigadores el punto al que Ilegaron los estudios latinoamericanos sobre la marginalidad, cuando llegan a conclusiones actuales sobre exclusión y pobreza? ${ }^{3}$

Entre innumerables colegas parecen regir los principios de incomunicabilidad, de constante desecho de ideas viejas no procesadas por ideas nuevas no procesadas, temas de estudio, y no verdadera búsqueda de problemas de investigación. Por lo demás, el nuestro es un subcontinente en que la vida social transcurre a velocidades difícilmente captadas por la investigación social, y los responsables de elaborar conocimiento se colocan por detrás de los cambios realmente existentes. Las razones tienen que ver con la pérdida de la memoria histórica, el conservadurismo y la soberbia académica, que desconoce o menosprecia conocimientos producidos por sus colegas en otras partes de la región, en otros períodos, o aún en sitios ajenos a sus propios centros de investigación. Tienen que ver también con los plazos que se fijan para la entrega de resultados, pero, sobre todo, con la dinámica de experiencias sociales intensas que aún testigos y participantes universitarios se muestran renuentes a recoger con amplitud en sus estudios.

\footnotetext{
3 Con todo, vale la pena destacar el esfuerzo que en cada uno de sus congresos ha realizado la Asociación Latinoamericana de Sociología por sintetizar críticamente las discusiones y debates en la región. Ver, por ejemplo, R.S., coord., América Latina y el Caribe: Perspectivas de su reconstrucción, México, ALAS, 1996; Emir Sader, ed, Democracia sin exclusiones ni excluidos, Caracas, Nueva Sociedad, 1998. Han contribuido a este esfuerzo, FLACSO, CLACSO y Nueva Sociedad, de la Fundación Friedrich Ebert. Véanse, por ejemplo, Fernando Mires, El discurso de la miseria o la crisis de la sociología en América Latina, Caracas, Nueva Sociedad, 1993; Alberto Acosta, El desarrollo en la globalización, Caracas, Nueva Sociedad, 2000; o Daniel Mato, comp., Estudios Latinoamericanos sobre cultura y transformaciones sociales en tiempos de globalización, Buenos Aires, CLACSO, 2001.
} 
No resulta fácil pensar, pensarse desde perspectivas distintas a las actuales, y las tentaciones para reproducir conocimientos socialmente considerados comunicables o asimilables desde la perspectiva dominante no dejan de ser fuertes. Existe, no obstante, una significativa tendencia a contestar en cada etapa la visión dominante, poniendo en cuestión sus afirmaciones, denunciando sus objetivos políticos o ideológicos y aún, poniéndose a distancia de los principios y criterios que postulan. Es esto lo que se ha comprendido como "pensamiento crítico" en diversos períodos, y puede decirse, sin temor a la equivocación, que la confrontación con el pensamiento dominante que sobre estas bases se realiza en toda América Latina constituye un principio significativo de construcción de conocimiento problemático y de comunicabilidad con pensamientos semejantes en nuestra propia región y en otras partes del mundo.

La perspectiva periférica, anticolonialista y antiimperialista se ha elaborado sobre el principio común de diferenciación respecto al conocimiento producido en los grandes centros de poder. Forma parte de la historia del más avanzado pensamiento latinoamericano, e indudablemente constituye la base sobre la que se funda nuestra identidad regional. Desde la Independencia hasta los inicios del siglo XXI, se ha mantenido vivo el interés de plantearse, desde distintas perspectivas, el significado, posibilidades y aún limitaciones de la unidad histórica problemática llamada América Latina. La propia denominación de Latinoamérica, Iberoamérica o América "a secas" ha formado parte de un debate que nos distingue de las definiciones primigenias de las ciencias sociales en otras regiones del mundo (una parte de las cuales, como sabemos, se han asumido históricamente sin escrúpulos como productoras de conocimientos "universales"). ${ }^{4}$ 
Con todo, el paso de la confrontación/diferenciación a la formulación de un pensamiento crítico no es simple. En el proceso, muchos conceptos y categorías pueden pasar a ser simplemente reciclados, si la intención no va más allá de buscar adecuarlos para lograr un posicionamiento en el debate. Por su parte, la formulación simple de temas o asuntos de interés (por más contemporáneos que sean) resulta limitativa e insuficiente. Se requiere, en cambio, construir campos problemáticos; replantearse a fondo los horizontes desde los cuales se visualizan dichos campos, así como las posibilidades que los sujetos sociales tienen de explotar las opciones que supone el conocimiento en la realidad.

Las preguntas comienzan, entonces, a ser otras. ¿Qué posibilidades abre al conocimiento la reconstrucción de experiencias semejantes vividas en sociedades regidas por códigos diversos? ¿Qué aportan la historia y la reflexión sobre una problemática en el conocimiento contemporáneo de la misma? ¿Qué sujetos han emprendido el conocimiento de dicha problemática, con qué objetivos, con qué alcances? ¿De qué elementos partimos para reconstruir una problemática determinada en nuestra propia sociedad? ¿Cómo construimos los instrumentos, las perspectivas adecuadas para enfrentar la tarea "desde abajo", "desde adentro"?

Nuestro ensayo se dirige a evaluar el peso de las orientaciones dominantes, como las perspectivas de construcción de un pensamiento crítico en la América Latina contemporánea. El ejercicio que realizaremos pudiera reproducirse, si se considera útil, en prácticamente cada campo de conocimiento social, previsto que se reconozca como tarea necesaria la de la elaboración de instrumentos de conocimiento que se sitúen más allá de las perspectivas establecidas por un pensamiento acotado y ahogado por la presión de incorporarse a la mayoritaria corriente de los reproductores de lo hecho por otros. 


\section{El territorio de las herencias: algunas armas de la crítica}

La determinación de políticas públicas -incluida la política de conocimiento social- por centros internacionales de poder, o lo que Edgardo Lander ha Ilamado la "colonialidad del saber" (Lander, 2000) coloca el empeño del conocimiento en un terreno difícil: la mayor parte de los centros de investigación y de docencia a nivel universitario tienden a reproducir no sólo los temas y tesis, sino los criterios de financiamiento impuestos por las corrientes hegemónicas internacionales, y, por tanto, las posibilidades de plantearse investigaciones en sentidos diversos a los considerados por ellas como pertinentes, son usualmente precarias.

Por lo demás, lo que se admite como parte de la estructura social determinada en buena medida por y en relación con el poder vigentefacilita el hecho de que las investigaciones se orienten de acuerdo a las perspectivas dominantes. Es más fácil, sugirió Sergio Bagú hace ya muchos años, conformarse con la observación de lo que en la sociedad aparece como "organizado" a partir de patrones establecidos por la cientificidad dominante. Parece una tarea en extremo delicada y difícil emprender el conocimiento de lo no contemplado o no reconocido por las rígidas estructuras categoriales dispuestas por dicha cientificidad, pese a que exista en la realidad (Bagú, 1970).

En la perspectiva de deconstruir las categorías y conceptos dominantes para aproximarse a un conocimiento que busque analizar críticamente nuestras sociedades desde lo que éstas efectivamente viven, Hugo Zemelman ha planteado la necesidad de captar la realidad en movimiento y en sus contradicciones fundamentales; identificar los sentidos, contenidos y perspectivas de cada experiencia histórica vivida desde quienes en ella intervienen; problematizar, no tematizar el conocimiento social (Zemelman, 1983). Su propuesta de analizar el movimiento contradictorio de lo real 
para romper con la estructura tradicional del pensamiento parece un empeño tan complejo como necesario, si de lo que se trata es de reconocer las condiciones en que objetivamente se configuran, articulan o confrontan los sujetos sociales en la historia.

A este asunto se dedicó también hace muchos años René Zavaleta. Reconstruir la experiencia y las perspectivas de la confrontación económica, social y política de los sujetos de nuestra historia; entenderla sometida a las tensiones y presiones de una construcción determinada por la condición de subalternidad, en horizontes de visibilidad cercados por poderes autoritarios, relaciones sociales atravesadas por contradicciones propias de tiempos distintos y superpuestos, voluntades fallidas, intentos inconclusos de resolver progresivamente sucesivas crisis. Su esfuerzo resta aún solitario y en gran medida, incomprendido en nuestros medios intelectuales. ${ }^{5}$

El recuento podría continuar, aunque brevemente, señalando a autores que han aportado conocimientos críticos acerca de cómo enfrentarse a la problemática latinoamericana, sin limitarse a reproducir lo elaborado en otras partes, desde el poder, desde el conformismo o aún desde perspectivas críticas rudimentarias, como las que señalamos en el apartado anterior. Por más de una razón -que en este trabajo no analizaremos-, el fascinante problema de construir una perspectiva propia resta aún, en gran medida, inexplorado en nuestra región. Del olvido y la premura por difundir resultados convincentes o "políticamente aceptables" están llenos, lamentablemente, nuestros centros de estudio.

No obstante, es posible considerar que la guía de pensadores que han recorrido la problemática histórica y contemporánea de nuestra región, particularmente de aquéllos que han definido a la región en su conjunto como objeto de estudio, y que se han planteado como problema principal

5 René Zavaleta, Lo nacional- popular en Bolivia, México, Siglo XXI, 1986, es expresión póstuma de su visión revolucionaria de la historia ... 
el de la identidad latinoamericana desde la que se realizan sus investigaciones es un punto de partida indispensable. El principio de analizar desde dónde, con quién, para quién, frente a quién se conoce es obligado para quien pretenda introducirse en la dinámica histórica y social latinoamericana.

\section{El territorio como tiempo y espacio social}

Uno de los problemas más complejos de enfrentar en el análisis social es el del territorio. Las aportaciones fundamentales de los geógrafos han sido poco incorporadas al conocimiento de la mayor parte de los científicos sociales, pero hemos de decir que, tan significativas como las suyas han pasado a ser, recientemente, las del pensamiento indígena en nuestro subcontinente $^{6}$ (Santos, 1997). En términos contemporáneos, pensar en el territorio implica reconocer no sólo una región física, lo que es de por sí difícil, ya que a ella se han impuesto criterios económicos y administrativos, sino ahondar en el terreno de la comunicabilidad e influencia del pensamiento, la presencia de la comunidad en espacios virtuales superiores a los que señala el espacio inmediato de referencia.

El territorio es y debe ser socialmente considerado, pues, en términos de su influencia intelectual, social y política, pero también -y sobre todo- en cuanto supone la existencia de una visión del mundo, es decir, un comportamiento histórica, social, cultural y políticamente orientado por encima de fronteras administrativas. El territorio es un espacio en el que se construyen y disputan relaciones sociales, formas de organización y esquemas de poder. Supone intensidad y durabilidad, pero también cambios en el tiempo. En este sentido, el territorio supone la inclusión del tiempo, entendido como sucesión de acontecimientos socialmente significativos, y por tanto, de los tiempos y ritmos de los sujetos que en ellos

6 Ver también Ediciones La Jornada, El otro jugador. La caravana de la dignidad indígena. México, 2001. 
participan. Es una forma de representación de las condiciones, contenidos, alcances de las relaciones sociales, y en particular, de las correlaciones de fuerzas y de la disputa por el poder en su área de influencia.

Identificar el territorio en que ocurre una problemática social es, entonces, una tarea indispensable para ubicarse en el conocimiento de la sociedad en referencia. Autores como Barrington Moore y James C. Scott han planteado este asunto en la perspectiva de la reconstrucción de códigos de comportamiento colectivo, incluidos los discursos ocultos, lo que constituye un punto de partida sugerente para abordar cualquier investigación social (Moore, 2000; Scott, 2000). Resta, sin embargo, plantearse un problema adicional, que en términos sociológicos se conoce como de la intensidad de las relaciones, y en términos históricos pudiera incorporar la categoría de la acumulación, es decir, la memoria socialmente vigente, la que se constituye en fuerza para orientar comportamientos sociales en sentidos determinados.

Aportaciones significativas en este terreno han sido realizadas por autores diversos, pero en América Latina señalaría fundamentalmente a René Zavaleta y Fernando Mires, quienes han realizado esfuerzos más sistemáticos por comprender las crisis como momentos y espacios socialmente significativos de intensidad y ruptura de las relaciones sociales previamente existentes; situaciones en que se produce una explosión de los horizontes de visibilidad de los sujetos y proyectos en conflicto en las sociedades latinoamericanas. Ellos han propuesto un método de conocimiento histórico basado en la identificación de los ritmos y aportaciones específicas de las crisis en el enfrentamiento y eventual superación de conflictos sociales (Mires, 1988; Zavaleta, 1974).

El conocimiento de las crisis y su sucesión en la historia contemporánea de América Latina puede y debe ser un instrumento esencial para identificar, en el momento de mayor intensidad, la calidad y orientación del 
comportamiento social, los ejes de la confrontación, la vigencia de un esquema de dominación en un territorio determinado. El conocimiento de cada crisis, su duración, los eventos que la desatan, los sujetos a los que involucra, el modo en que se resuelve, la cualidad por la que se relaciona con crisis previas y anticipa crisis futuras, constituyen valiosos instrumentos de identificación del tiempo y el espacio, los códigos, las representaciones, las posibilidades y los condicionamientos de las relaciones sociales, de la voluntad y direccionalidad de los sujetos que intervienen en cada crisis y del desarrollo de la sociedad en su conjunto.

\section{El territorio de lo realmente existente}

Por las razones expuestas, es evidente que, hasta ahora, hemos sido en gran medida incapaces de articular, de manera sostenida, la potencialidad crítica que nuestros más avanzados pensadores han logrado en la historia contemporánea de la región. El panorama actual dista de la solidez a que debiéramos aspirar, si pudiéramos seguir las líneas planteadas por nuestra propia experiencia intelectual como latinoamericanos.

La mayor parte de nuestros trabajos tiende, en efecto, a construirse desde una de las siguientes y limitadas perspectivas:

La reproducción acrítica de modelos y categorías de pensamiento elaborados desde los centros de poder, y que se pretenden no sólo hegemónicos, sino únicos y virtualmente capaces de explicar todas las realidades. Lo aportado por esta orientación suele limitarse -en el mejor de los casos- a señalar los grados de aproximación de una expresión determinada de la vida social a la tendencia "universal" 
(básicamente, a la concebida como tal en los centros de poder);

La constatación crítica de los efectos que la imposición de políticas y normas de conocimiento tiene sobre la vida pública. Lo aportado por esta perspectiva se sitúa en el campo de la denuncia, en la diferenciación de lo impuesto respecto de necesidades insatisfechas de la sociedad (en los últimos tiempos, señaladamente, entendido como ejercicio del derecho a la vida, la educación, la salud, el respeto a la integridad de los seres humanos, el reconocimiento y tolerancia a la diversidad);

El reconocimiento de una problemática social específica, usualmente promovido por estudios empíricos sobre la evolución de fenómenos y, eventualmente, su impacto en el conjunto de la vida social. Lo aportado por esta perspectiva se sitúa, en todos los campos de la vida social, en el conocimiento de procesos, mediante su cuantificación, la construcción de indicadores de impacto, la contextualización de eventos por su seguimiento cotidiano o sistemático;

La búsqueda de alternativas de superación a las problemáticas sociales reconocidas. Estas pueden plantearse, y usualmente lo hacen, en uno de dos planos: el de la ubicación de tendencias, entendidas como reconstrucción de experiencias en el señalamiento de caminos hacia el futuro; o el de la más modesta constatación de efectos previsibles ante el agravamiento de fenómenos diversos, continuación de enfrentamientos o polarizaciones en otros planos, etc. Una parte de estos trabajos se sitúan más en el plano ideológico (y hasta podría decirse, pedagógico), de promoción de ideas, valores, intenciones o voluntades, y otra, inevitablemente se aproxima a la segunda perspectiva señalada, que es la de la constatación 
crítica de eventos, procesos, políticas.

En la medida en que estas perspectivas tienden a convertirse - O son ya-corrientes de pensamiento desarticuladas o confrontadas en el estudio de problemas sociales reales y actuales en América Latina, conviene realizar un ejercicio de revisión, aunque sea inicial y somero, del lugar en que nos encontramos, para emprender nuevas búsquedas y aproximarnos de manera menos incierta al conocimiento y transformación de nuestra realidad.

Para los efectos de esta revisión, denominaremos a la primera orientación esbozada, de reproducción acrítica; a la segunda, de denuncia; a la tercera, de constatación empírica; y a la última, de replanteamiento de los estudios latinoamericanos. Es evidente que esta diferenciación sólo opera parcialmente, ya que suele ser pretensión -reconocida o no- de los estudiosos construir y difundir su propia visión de los hechos sociales como antecedente o guía para intervenir en su curso.

\section{Territorios de la representación del poder}

La divulgación de temas socialmente aceptados en nuestra región, considerada territorialmente, ha estado sobre todo a cargo de las agencias internacionales de financiamiento económico. Estas dominan la construcción de la estadística social y la determinación de las orientaciones de estudio a partir de dos asunciones fundamentales: la sociedad debe medirse en función de la evolución cuantitativa de indicadores individuales prestablecidos (el empleo, el ingreso, el acceso a la salud o a la educación, el crecimiento económico, etc.), y no puede rebasar los marcos de determinaciones "universales" de validación.

Los cortes de conocimiento que se establecen a partir de esta versión tematizada suponen, desde luego, que es posible atravesar las fronteras nacionales en la perspectiva de una homogeneización internacional, pero sobre todo, que cualquier espacio social es susceptible de fragmentación, 
mientras que se considera inválido todo cuestionamiento sistémico. Asumir la validez universal de tal perspectiva significa no sólo negar las especificidades de cada realidad social, sino ubicar el conocimiento desde el territorio virtual de los centros de poder internacional, para los cuales el impacto de sus orientaciones y políticas en comunidades determinadas es asunto secundario o no considerado en absoluto.

Esta operación de fragmentar y cuantificar el conocimiento social no sólo supone la deshumanización en el abordaje de problemas sociales graves, sino el desconocimiento de las características y condiciones en que se implanta cada política en sociedades con historia, realidades culturales, necesidades y agravios determinados. Tematizar el conocimiento de la realidad social a partir de esta visión significa, de entrada, excluir lo real no reconocido por los parámetros que supone. Diversos autores han hecho ejercicios críticos sobre el conocimiento de problemáticas sociales, en particular, el desarrollo, la pobreza y la violencia en Latinoamérica, partiendo de la crítica a esta visión (Sader; Gentili, 1999; Sader, 2001). Asimismo, debe realizarse en relación a los ejes de la confrontación internacional, como lo han planteado, entre otros, Noam Chomsky y John SaxeFernández (Chomsky, 2002; Saxe-Fernández, 1999).

El hecho socialmente significativo es que la existencia misma de esta orientación del pensamiento nos obliga a realizar una revisión a fondo del modo en que se ha incorporado en la dinámica de la confrontación en cada territorio. Y en vista a su influencia, debe considerarse el impacto de la negación del espacio y el tiempo de las relaciones sociales específicas en la orientación de los sujetos asociados al esquema impuesto de dominación.

\section{Los territorios de la resistencia}

Pocos trabajos dirigidos a denunciar la perspectiva dominante se ocupan de penetrar el territorio de su influencia en la construcción categorial de 
investigaciones y políticas sociales, incluyendo no sólo en los espacios públicos, sino en centros independientes de estudio y hasta organizaciones civiles. Ya hace muchos años, la polémica desatada por James Petras en su análisis crítico de los intelectuales orgánicos a las dictaduras latinoamericanas ocupó espacios significativos del debate sociológico y contribuyó a la revisión de las orientaciones del pensamiento en la región (Petras, 1988; Vilas, 1990). El asunto, con todo, sigue estando presente, sólo que ahora se plantea en términos de la aceptación acrítica de conceptos, categorías, temas de estudio y orientaciones, sin que se desarrollen intentos de deconstrucción crítica de los elementos en que se sostiene el pensamiento dominante (lanni, 1997).

La utilización de una estadística social construida a partir del esquema de pensamiento impuesto por centros internacionales de poder es uno de los ejemplos fehacientes de cómo es posible recurrir a instrumentos presuntamente neutrales para ocultar la realidad, para orientar el conocimiento hacia los parámetros aceptados. Véanse, si no, las estadísticas sobre pobreza y empleo, e inténtese reconstruir a partir de ellas una visión de lo que realmente ocurre en nuestras sociedades en esa materia. La distancia se hace aún mayor si de lo que se trata es de interpretar el impacto de las políticas públicas en la superación de los llamados rezagos sociales. En el primer caso, la conclusión debiera ser que el perfil de un pobre, desempleado o subempleado es irreconocible, como lo es la cadena social que alimenta la reproducción de la pobreza. Piénsese, por ejemplo, en las diferencias entre un pobre urbano y un pobre rural, en las condiciones del llamado empleo informal o, incluso, en el peso de la migración interna y externa. Ninguna de estas presencias reales está actualmente reconocida por una estadística que se limita a recuentos superficiales, ordenados de acuerdo a criterios ajenos a la dinámica social objetiva, más allá de la alarma que produzca su volumen. ¿Qué significa, después de todo, que existan 460 
millones de pobres en la región?

Si intentáramos reconocer en la estadística las confrontaciones políticas, los proyectos de poder, el peso de la economía ilegal -el narcotráfico, la venta de armas-, la intervención externa, la tarea se hace imposible. ¿Dónde, cómo se ubican treinta mil millones de dólares anuales en operaciones de narcotráfico en el mundo? ¿Qué peso específico ha tenido en los últimos cinco años la venta de más de 100,000 millones de dólares en armamentos? ¿Qué relaciones de poder se construyen en cada país y a nivel internacional a partir de este comercio?

Replantear la estadística social, construir formas de cuantificación en que se reconozcan e identifiquen proyectos y sujetos sociales, normas y formas de supervivencia, dinámicas colectivas, resta como un terreno esencial para la construcción de un pensamiento crítico más allá de la denuncia en los términos esperados y acotados por el pensamiento dominante. Ir más allá del deslinde de políticas públicas ineficaces o equívocamente orientadas si de lo que se tratara es de superar problemas sociales; más allá aún de la confrontación de cifras construidas en función de modelos de sociedad impuestos, hasta el reencuentro con los seres humanos, con su dignidad, carencias y demandas, puede ser el inicio de una reformulación verdadera del conocimiento social.

La tarea, con todo, no es sólo de reconstrucción estadística, sino de replanteamiento del uso en general de fuentes de información, lo que incluye no sólo un trabajo transdisciplinario en la identificación de los territorios involucrados en cada objeto de estudio, sino la construcción de una metodología que nos permita captar el movimiento en el tiempo y en el espacio, la actualización de la memoria, la ocurrencia de fenómenos nuevos o no previamente estudiados, la dinámica de relaciones sociales, el escalamiento o desescalamiento de conflictos, los cambios en la estructura 
del poder.

En la realización de este empeño, contribuyen de manera significativa tanto las constataciones empíricas de hechos, como los esfuerzos encaminados en sentido alternativo, o de replanteamiento de los estudios. Unas y otros, con todo y vacíos o ausencias, apuntan en el camino de una reorientación metodológica del trabajo de investigación, siempre y cuando nos permitan acercarnos a la reconstrucción de los territorios sociales, analizados en su complejidad, como en cuanto a lo que del pasado se expresa en el presente, lo que del presente se anuncia en condición de escenario o tendencia hacia el futuro.

La hipótesis de ejes de conflicto, de la existencia de estructuras y sujetos sociales y de poder debe ser puesta a prueba en términos del descubrimiento de elementos que definan la comunicación, la intensidad de relaciones, el movimiento de los sujetos en el tiempo y en el espacio. Así, cualquier problema de investigación puede convertirse en espacio de interrogación sobre la dinámica social, en momento de reconocimiento de posibilidades de ampliación del horizonte de visibilidad de los sujetos en un proceso social, y hasta de construcción utópica. El conocimiento social, en definitiva, puede así reconocer- y hacer aportaciones sociales para enfrentar- las dificultades y obstáculos que atraviesan los sujetos en sus aspiraciones y voluntad práctica de transformar sus condiciones de vida y a su sociedad. Replantear, reconstruir los instrumentos y métodos del conocimiento con ese dimensionamiento del tiempo y el espacio, de sujetos y territorios, es, indudablemente, un reto contemporáneo formidable.

\section{Referências}

BAGÚ, Sergio. Tiempo, realidad social y conocimiento. México: Siglo XXI, 1970. 
CHOMSKY, Noam. En La Jornada. México: La Jornada Ediciones, 2002.

IANNI, Octavio. As ciências sociais na época da globalização. Estudios Latinoamericanos, México, Nueva Época, Año IV, n. 8, julio-diciembre. 1997.

LANDER, E. (comp.). La colonialidad del saber: eurocentrismo y ciencias sociales. Perspectivas latinoamericanas. Caracas: UCV-UNESCO, 2000.

MIRES, Fernando. La rebelión permanente. Las revoluciones sociales en América Latina. México: Siglo XXI, 1988.

MOORE, Barrington. La Injusticia: bases sociales de la obediencia y la rebelión, México: IIS-UNAM, 1989.

PETRAS, James. La metamorfosis de los intelectuales latinoamericanos. Estudios Latinoamericanos, México, n. 5, CELA-FCPS-UNAM, 1988.

SADER, Emir (comp.). El ajuste estructural en América Latina: costos sociales y alternativas. Buenos Aires: CLACSO, 2001.

SADER, Emir y GENTILI, Pablo. La trama del neoliberalismo. Mercado, crisis y exclusión social. Buenos Aires: CLACSO/Eudeba, 1999.

SANTOS, Milton. A natureza do espaço. Técnica e tempo. Razão e emoção. São Paulo: Hucitec, 1997.

SAXE-FERNÁNDEZ, John. Globalización: crítica a un paradigma. México: UNAM/ Plaza y Janés, 1999.

SCOTT, James C.. Los dominados y el arte de la resistencia. México: Era, 2000.

VILAS, Carlos. Intelectuales, dólares y compromiso: un comentario a James Petras. Estudios Latioamericanos, México, n. 8, CELA-FCPS-UNAM, 1990.

ZAVALETA, René. Movimiento obrero y ciencia social. Historia y Sociedad, MéxiCo, n.3, otoño. 1974.

ZEMELMAN, Hugo. Historia y política en el conocimiento. México: FCPS-UNAM, 1983.

Recebido: 01/03/2005

Aceite final: 15/04/2005 


\section{Resumo}

En América Latina, el estudio de las determinaciones sociales del conocimiento, o lo que pudiera llamarse una sociología de los modos de pensar, es poco frecuente. Podemos, sin temor a equivocarnos, asumir que se trata de un campo prácticamente inexplorado.

El nuestro es un subcontinente en que la vida social transcurre a velocidades difícilmente captadas por la investigación social, y los responsables de elaborar conocimiento se colocan por detrás de los cambios realmente existentes. Las razones tienen que ver con la pérdida de la memoria histórica, el conservadurismo y la soberbia académica, que desconoce o menosprecia conocimientos producidos por sus colegas en otras partes de la región, en otros períodos, o aún en sitios ajenos a sus propios centros de investigación. Tienen que ver también con los plazos que se fijan para la entrega de resultados, pero, sobre todo, con la dinámica de experiencias sociales intensas que aún testigos y participantes universitarios se muestran renuentes a recoger con amplitud en sus estudios.

Nuestro ensayo se dirige a evaluar el peso de las orientaciones dominantes, como las perspectivas de construcción de un pensamiento crítico en la América Latina contemporánea. El ejercicio que realizaremos pudiera reproducirse, si se considera útil, en prácticamente cada campo de conocimiento social, previsto que se reconozca como tarea necesaria la de la elaboración de instrumentos de conocimiento que se sitúen más allá de las perspectivas establecidas por un pensamiento acotado y ahogado por la presión de incorporarse a la mayoritaria corriente de los reproductores de lo hecho por otros.

Palabras-clave: colonialidd, subalternidad, dominación, pensamiento crítico, construcción de conocimiento problemático. 


\section{Legacies of and future directions for knowledge in Latin America}

Raquel Sosa Elízaga

In Latin America, the study of social determinations of knowledge, or what we could call a sociology of ways of thinking, is not common. We can, without mistaking it, assume that such field is virtually unexplored. Life in our subcontinent develops at paces that are hardly captured by social investigation, and those responsible for producing knowledge stay behind really existing changes. The reasons are related to the loss of historical memory, the conservatism and academic haughtiness, which ignores or downplays knowledge produced by their colleagues in other parts of the region, in other times, or also in places that are distant from their own investigation centers. They are also related with the deadlines set for delivering results, but, most of all, to the dynamics of intense social experiences that university witnesses and participants are still reluctant to broadly collect in their studies.

Our essay is aimed at evaluating the weight of predominant orientations, such as the perspectives of the construction of a critical thinking in contemporary Latin America. The exercise we carry out can be reproduced - if it is useful - in virtually every field of social knowledge, as long as it necessarily includes the task of producing knowledge tools that go beyond the perspectives established by a way of thinking tormented and suffocated by the pressure to be incorporated into the majority group of reproducers of what others have done.

Key words: coloniality, subalternity, domination, critical thinking, construction of problematic thinking. 